\title{
Early HIV infection: to treat or not to treat?
}

\section{Cautiously, until trials produce answers on long term efficacy and safety}

In August, following the recommendation from the European committee on proprietary medicinal products, the Department of Health extended the product licence for zidovudine to include patients infected with HIV who have early symptomatic disease or who are asymptomatic but have markers of progressive disease. The risk markers include repeated peripheral blood CD4 + (T helper) lymphocyte counts of less than $2 \times 10^{9} / 1$ or counts between 2 and $5 \times 10^{9} / 1$ that are falling rapidly. The data that have influenced these changes were obtained from studies in the United States by the AIDS Clinical Trials Group (ACTG 019, 016, and 002).

The first of these studies (ACTG 019) compared high dose zidovudine (1500 mg/day), low dose zidovudine (500 $\mathrm{mg} /$ day), and placebo in asymptomatic patients to determine its safety and efficacy in delaying progression to symptomatic disease and prolonging survival. ${ }^{1}$ Several problems were encountered that reflect both rapidly changing clinical practice and the climate in which trials of HIV infection are now performed. Although the study was intended to be double blind, doctors and patients were not blinded to patients' mean corpuscular volumes - which zidovudine increases. Some patients allocated to placebo took zidovudine, there were anecdotal reports of drug sharing, and the placebo group had a much higher level of default and voluntary withdrawal than the zidovudine group. Nevertheless, the trial advisers thought that these problems were unlikely to lead to unacceptably large biases.

The progression rate to AIDS and advanced AIDS related complex in patients with baseline CD4+ cell counts less than $5 \times 10^{9} / 1$ was about halved from $7 \cdot 6$ per 100 person years in the placebo group to 3.6 and 4.3 per 100 person years in the low and high dose groups respectively. Few people benefited, however, and the mean follow up was only 55 weeks. Severe anaemia (haemoglobin concentration less than $8.0 \mathrm{~g} / \mathrm{l}$ ) occurred in $6.3 \%$ of those taking the high dose but only $1 \cdot 1 \%$ of those taking the low dose and was the main reason for the independent data and safety monitoring board's recommendation of the $500 \mathrm{mg}$ dose for open zidovudine treatment in people with initial CD4+ cell counts less than $5 \times 10^{9} / 1$.

The next trial (ACTG 016) compared zidovudine (1200 mg/ day) with placebo in early symptomatic disease. The clinical end points were AIDS, advanced AIDS related complex, and death. ${ }^{2}$ In a mean follow up of nine months 21 of 351 patients in the placebo group and 5 of 360 in the zidovudine group developed AIDS as the first critical event, with an overall event rate (progression to AIDS or advanced AIDS related complex) of $11 \cdot 2$ per 100 person years for the placebo group and 4.5 per 100 person years for the zidovudine group. Nevertheless, only five events occurred in patients with initial CD $4+$ cell counts of over $5 \times 10^{9} / 1$. Severe anaemia occurred in $5 \%$ of the zidovudine group but in none of the placebo group.

The third study (ACTG 002) compared two doses of zidovudine, $1500 \mathrm{mg} /$ day or $1200 \mathrm{mg} /$ day for one month followed by $600 \mathrm{mg} /$ day in patients who had had one episode of Pneumocystis carinii pneumonia (M A Fischl et al, sixth international conference on AIDS, San Francisco, 1990). In a median follow up of 26 months the numbers of new opportunistic infections, malignancies, and deaths and changes in p24 antigen concentrations and CD4+ cell counts were similar in both groups. There was, however, a substantially higher incidence of haematological toxicity in the high dose arm; in fact this group received on average a smaller amount of the drug than the low dose group. Low dose zidovudine results in less toxicity, but the two doses may not be equally effective; a non-significant result does not necessarily imply equivalence.

The extension of zidovudine's product licence to asymptomatic people is based on evidence of acceptable toxicity and efficacy. The trials so far, however, have reported only short term results and have not determined whether early intervention prevents long term progression of disease and improves survival. Extended clinical experience suggests that in patients with symptomatic HIV disease the benefit of zidovudine does not last indefinitely. ${ }^{3}$

Viral resistance to zidovudine has been shown in most isolates from patients with symptomatic disease treated with zidovudine for six months or more. ${ }^{4}$ In asymptomatic people resistant strains seem to emerge more slowly and, so far, with a lower level of resistance ( $M$ H St Clair et al, C A B Boucher et al, sixth international conference on AIDS, San Francisco, 1990). What happens to patients who receive zidovudine for several years when they are well and then subsequently progress to symptomatic disease remains to be seen. Their survival after this might well be reduced.

The optimum dose of zidovudine is not established. A daily dose of $1500 \mathrm{mg}$ produces too many toxic effects when compared with $500 \mathrm{mg}$ or $600 \mathrm{mg}$. But the efficacy of the lower doses, particularly in treating or preventing disease of the central nervous system, and their relation to the development of resistance is unknown. In Europe, where the standard initial daily dose and that in current trials is $1 \mathrm{~g}$, many doctors may be reluctant to change their clinical practice.

Treating asymptomatic people also brings inevitable 
psychosocial consequences. They need to attend clinics and have regular blood tests. Furthermore, minor toxicity can affect the quality of life, particularly if the drug is to be taken for many years by those who would otherwise be well.

Zidovudine is still the only antiviral drug available with proved efficacy in HIV disease, but the extension of its licence to asymptomatic disease does not make the decision of when to start treatment any easier for the doctor or the patient. A decision based solely on the results of a laboratory investigation that is not universally available and whose biological and laboratory variability is considerable is clearly difficult. ${ }^{5}$ The terms "repeated" and "rapidly falling" in relation to CD4+ cell counts are not defined clearly because the data are insufficient. The European and Australian trials in asymptomatic people must continue to answer fully the long term questions about efficacy and toxicity. Any move to intervene in asymptomatic infection and to prescribe low doses should be approached with caution.

ANN MARIE SWART Lecturer

IAN WELLER

Reader in Genitourinary Medicine University College and Middlesex School of Medicine,

Middlesex Hospital,

London W1N 8AA

Department of Clinical Epidemiology

National Heart and Lung Institute,

Royal Brompton and National Heart Hospital,

London SW3 6HP

1 Volberding PA, Lagakos SW, Koch MA, et al. Zidovudine in asymptomatic human immunodeficiency virus infection. A controlled trial in persons with fewer than $500 \mathrm{CD} 4$-positive cells per cubic millimeter. N Engl f Med 1990;322:941-9.

2 Fischl MA, Richman DD, Hansen N, et al. The safety and efficacy of zidovudine (AZT) in the treatment of subjects with mildly symptomatic human immunodeficiency virus type 1 (HIV) treatment of subjects with mildy symptomatic human immunodeficiency virus type

3 Fischl MA, Richman DD, Causey DM, et al. Prolonged zidovudine therapy in patients with AIDS and advanced AIDS-related complex. FAMA 1989;262:2405-10.

4 Larder BA, Darby G, Richman DD. HIV reduced sensitivity to zidovudine (AZT) isolated during prolonged therapy. Science 1989;243:1731-4

Bird G. Monitoring of lymphocyte sub-population changes in the assessment of HIV infection. Genitourin Med 1990;66:133-7.

\section{Endemic bladder stones}

\section{Now disappeared from developed countries but still commonplace in much of Asia}

From antiquity to the early twentieth century Europeans were prone to bladder stones. Along with circumcision and trephining their removal is one of the three oldest surgical procedures.' Bladder stones remained common in the nineteenth century within certain "stone districts," such as Norfolk, where itinerant lithotomists flourished, and in London "cutting for the stone" was the commonest operation at the Westminster Hospital. ${ }^{2}$ Suddenly they disappeared, so that by 1957 Myers could write that only one child with a primary bladder stone had been seen at the Hospital for Sick Children, Great Ormond Street, in the preceding 20 years. ${ }^{3}$

The cause of this once common condition was probably dietary. Although the poor, particularly the urban poor, were most susceptible, malnutrition did not seem to be the cause. Several theories have implicated the cereal composition of the diet. $^{+5}$ In an ingenious study Halstead noted a negative correlation between the number of cows per head of population and hospital admissions for bladder stones throughout nineteenth century England and suggested that in areas where milk was short infants would be fed "pap"- bread and water. ${ }^{6}$
This diet of a single cereal (wheat) was implicated as the cause of bladder stone formation. The subsequent decline in bladder stones coincided with the advent of the milk train and pasteurisation, both of which brought milk to the urban poor, reducing their dependence on pap. The recent hypothesis that a low calcium intake also encourages bladder stone formation may further explain why the increased availability of milk hastened their disappearance. ${ }^{\text {? }}$

Although they have disappeared in developed countries, bladder stones remain an important cause of morbidity in a belt extending from the Middle East across India and Thailand to Indonesia. In this endemic area cystolithotomy accounts for about $10 \%$ of surgical operations in children ${ }^{78}$ and in some pockets of high endemicity in Thailand up to half of all non-obstetric surgery. ${ }^{9}$ Within this belt the urban middle classes are invariably spared. Just as in nineteenth century England, a diet based on a single cereal seems to be the most important aetiological factor, whether this is wheat ("bulgur" in Turkey), rice, or millet. ${ }^{5}$ Maize, however, the staple food in much of Africa, ${ }^{10}$ does not seem to be implicated, and South Americans also seem to be curiously exempt, possibly because of a more mixed cereal component to their diet (S Ismail, personal communication). ${ }^{11}$

Endemic stones from both the bladder stone belt and English collections 80-200 years old consist of ammonium acid urate and calcium oxalate. ${ }^{12}$ These contrast with those composed of phosphates and calcium oxalate, which are still seen in developed countries and result from urinary obstruction or infection. Ammonium acid urate is probably the more important substance in the nucleation of endemic stones, ${ }^{12}$ its precipitation requiring high urinary concentrations of both uric acid (which is present in all normal children, falling to adult concentrations by the age of 10) and ammonia. The diet may favour precipitation of ammonium acid urate in several ways. Firstly, a single cereal diet of rice in infants raises the urinary ammonia concentration. ${ }^{13}$ Secondly, a diet low in calcium, such as one of rice, little or no milk, and soft water, would result in hypocalciuria, which facilitates precipitation of ammonium acid urate in vitro. ${ }^{1+}$ Thirdly, urinary pyrophosphates act as non-specific inhibitors of precipitation, ${ }^{15}$ and their presence is promoted by dietary phosphate, again found in milk. Although certain foods raise the urinary uric acid concentration, it is not clear if they play an important role in formation of ammonium acid urate stones. Dietary factors that influence the precipitation of the other major component of endemic stones, calcium oxalate, are less well defined.

Does the single cereal theory alone explain the variations in the prevalence of bladder stones within the Asian bladder stone belt and their absence in South America and Africa? Final proof of the single cereal theory is lacking. This will depend on evidence that the essential prerequisite, a raised urinary ammonia concentration in infants, can be caused by each of the implicated cereals when eaten singly and is not caused by maize (thus explaining the absence of stones in Africa) or by a mixed cereal diet. So far, no studies have compared urinary ammonia concentrations in areas of high and low prevalence. On theoretical grounds milk may have a protective role against the development of bladder stones, ${ }^{7+1}$ but no studies have examined whether communities in which weaning is late experience fewer stones. Conversely, early weaning, often on the first day after birth, is the custom within one small pocket in Thailand where bladder stones are hyperendemic, ${ }^{16}$ but it is not known whether other communities in which neonates are weaned are more susceptible. Nevertheless, given the large variation in Asian weaning practices, the age of weaning is unlikely to explain the unique existence of a stone belt. Hard water, which can contribute a 